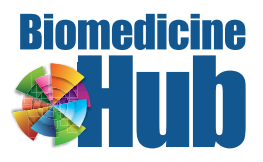

\title{
A Sterile Collection Bundle Intervention Reduces the Recovery of Bacteria from Neonatal Blood Culture
}

\author{
Linze F. Hamilton ${ }^{a}$ Helen E. Gillett ${ }^{a}$ Adam Smith-Collins ${ }^{a}{ }^{a}$ \\ Jonathan W. Davis ${ }^{c, d}$ \\ ${ }^{a}$ Regional Neonatal Intensive Care Unit, St. Michael's Hospital, University Hospitals Bristol \\ NHS Foundation Trust, Bristol, UK; ${ }^{b}$ Neonatal Neuroscience, University of Bristol, Bristol, \\ UK; ' ${ }^{C}$ Neonatal Clinical Care Unit, King Edward Memorial and Princess Margaret Hospitals, \\ Perth, WA, Australia; ${ }^{d}$ Centre for Neonatal Research and Education, University of Western \\ Australia, Perth, WA, Australia
}

\begin{abstract}
What Is It about?
Babies in intensive care are at risk of infection. The main organism causing this is a member of the Staphylococcus family and is found commonly on human skin. Blood cultures are easily contaminated by this organism. It is often difficult to decide if these bacteria are in blood culture and whether the patient has a true infection. We conducted a study to determine whether a sterile technique to acquire blood cultures would reduce the number of false-positive results.
\end{abstract}

\section{Keywords}

Blood culture $\cdot$ Neonate $\cdot$ Sterile collection $\cdot$ False positives $\cdot$ Coagulase-negative Staphylococcus

\section{Abstract}

Background: In neonatal intensive care, coagulase-negative Staphylococcus species can be both blood culture contaminants and pathogens. False-positive cultures can result in clinical uncertainty and unnecessary antibiotic use. Objective: This study sought to assess whether a sterile blood culture collection bundle would reduce the incidence of false-positive blood cultures in a regional neonatal intensive care unit. Method: Clinical data was collected from all infants who had blood cultures taken before and after the introduction of the sterile blood culture collection bundle intervention. This intervention required $2 \%$ chlorhexidine and full sterile precautions for blood culture collection. False-positive blood culture rates (presence

H. Gillet and L. Hamilton contributed equally to the preparation of the manuscript as first authors. 
of skin commensals and $\geq 3$ clinical infection signs) were compared before and after the intervention. The number of days of unnecessary antibiotics associated with false-positive blood cultures was also analysed. Results: In the pre-intervention group (PRE) 197 cultures were taken from 161 babies. In the post-intervention group (POST) 170 cultures from 133 babies were acquired. Baseline demographics were similar in both groups. The rate of false-positive cultures in the PRE group versus the POST group was 9/197 (4.6\%) compared to 1/170 (0.6\%) $(p<0.05)$. Unnecessary antibiotic exposure was reduced in the PRE group in comparison to the POST group ( 27 vs. 0 days, $p<0.01$ ). Conclusions: Implementation of sterile blood culture collection intervention reduced the number of false-positive results. This has potential benefit in reducing unnecessary antibiotic use.

(C) 2018 The Author(s)

Published by S. Karger AG, Basel

\section{Background and Aim}

Infection has a significant impact on the morbidity and mortality of newborn infants. The NeonIN Surveillance Network in England (data from 2007 to 2008) has reported a total incidence for all neonatal infections of 8/1,000 live births [1]. Late-onset infections increase the likelihood of cerebral palsy and poor neurodevelopmental outcome in preterm low-birthweight infants [2]. It is therefore extremely important to identify the source of any infection accurately and treat promptly [3].

In preterm infants, coagulase-negative Staphylococcus (CoNS) is a major cause of lateonset sepsis. The highest proportion of CoNS infections are in infants $<32$ weeks [4]. When CoNS is identified in a blood culture, it is difficult to determine whether its growth represents a true blood stream infection or contamination from skin flora [5]. This leads to clinical uncertainty $[4,6]$ and potentially prolongs treatment with antibiotics, lengthens stay, and increases hospital costs [7].

There is wide variation in contamination rates between populations. In the adult inpatient population in the USA, blood culture contamination is reported to be between 0.6 and $6 \%$ [3]. In a retrospective study of 9,959 blood cultures performed in children aged 1 month to 18 years [8], $26 \%$ of children had a false-positive culture. Contaminated blood cultures occur frequently in the neonatal population with reported rates between 2.6 and $18 \%[9,10]$. This is felt to be due to the difficulty in obtaining blood samples [4, 9].

There are several published methods to reduce blood culture contamination in the paediatric and neonatal population. In the paediatric population, Hall et al. [11] showed a reduction in contamination rates by using sterile gloves and a sterile field for blood culture collection in the setting of a paediatric emergency department. Other studies have improved contamination rates by using different skin cleansing solutions [11,12], dedicated phlebotomy teams [13], commercially produced blood culture collection packs [14], staff education programs [15], and by improvements in hand hygiene [16]. Despite these measures, CoNS-positive blood cultures remain problematic for the physician and infant. Our study aimed to assess the impact of a blood culture collection bundle on the incidence of false-positive (contaminated) blood cultures in a regional neonatal intensive care unit.

\section{Method}

This study was a single-centre non-randomised intervention study in the neonatal intensive care unit in St. Michael's Hospital in Bristol, UK, which is part of the University Hospitals Bristol NHS Foundation Trust. St. Michael's neonatal unit has approximately 800 
Table 1. Description of the sterile collection bundle intervention

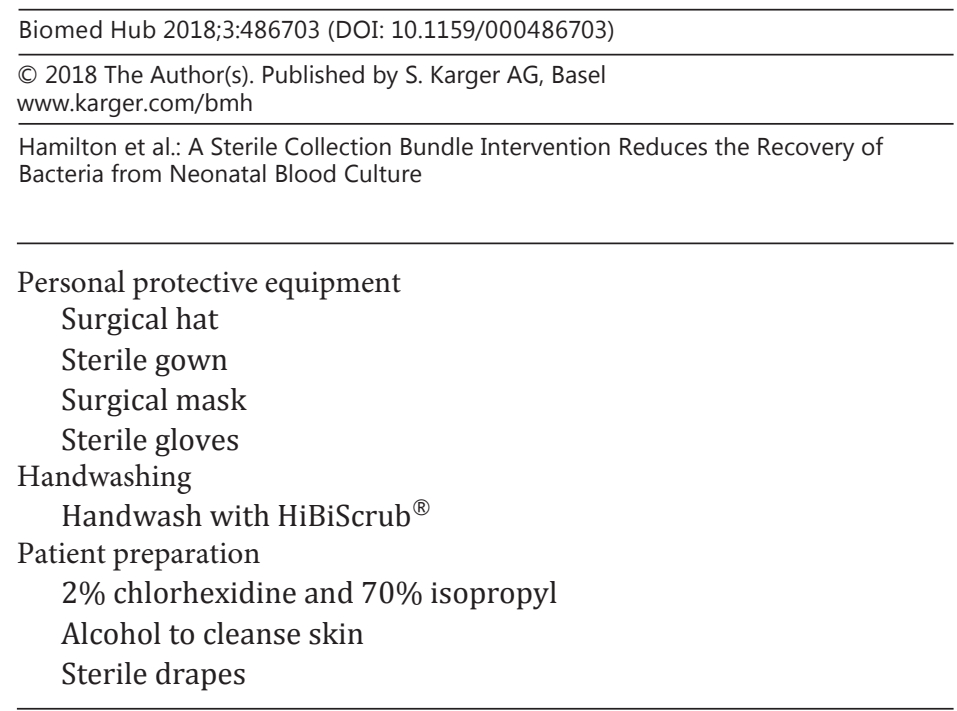

admissions and supports 6,000 deliveries per year. It is the regional referral centre for neonatal surgery, cardiology, and therapeutic hypothermia, and receives referrals from Southwest England and South Wales.

The study included infants born in St. Michael's hospital who required a blood culture for the investigation of sepsis between October 2012 and May 2013. Two time epochs were assessed: one before and one after the intervention in March 2013, i.e. October to November 2012 (PRE) and April to May 2013 (POST). The intervention was assessed a second time in June/July 2017 for sustained efficacy. The PRE epoch served as a control period. During this time, the standard practice in the neonatal unit was to collect blood cultures using an aseptic non-touch technique. This consisted of hand cleansing with soap (Diversey H21, Diversity Ltd., Northampton, UK) applying non-sterile gloves, cleansing of the patient's skin with $0.05 \%$ chlorhexidine, and thereafter avoiding touching the patient's skin.

In March 2013, the sterile blood culture collection bundle intervention was introduced. This comprised preparing the patient's skin with $2 \%$ chlorhexidine gluconate (ChG) and $70 \%$ isopropyl alcohol (ChloraPrep ${ }^{\circledR}$, CareFusion, USA) hand cleansing with HiBiScrub ${ }^{\circledR}$ (Mölnlycke Health Care, Sweden), and full aseptic barrier precautions including the use of a sterile gown, gloves, hat, and field (Table 1).

The sterile collection bundle intervention was introduced after approval was given by the University Hospitals Bristol Foundation Trust Governance Committee. A face-to-face explanation was given to all doctors and advanced neonatal nurse practitioners as to the requirements and reasoning behind this introduction. The clinical team was not aware that the intervention was being assessed to detect effectiveness.

Blood cultures were collected in a paediatric blood culture bottle (BacT/ALERT ${ }^{\circledR}$, Biomérieux Inc., Craponne, France). They were incubated for 5 days and were classified as positive if an organism was grown during this time. The recommended volume of blood collected for culture was $1 \mathrm{~mL}$ [17]. Data regarding clinical status and results were obtained from medical notes, nursing charts, electronic medical records database, and laboratory results. The data collected included demographics of sex, weight, and gestation; maternal risk factors for infection; clinical indicators of infection; blood culture result and type; and duration of antibiotics.

Positive blood cultures were scrutinised and subdivided into true and false positives using a definition developed by Modi et al. [4]. This definition was developed to assist in defining true blood stream infection in the neonatal period. Using this definition, a blood culture is considered to be a true blood stream infection if the bacteria isolated are a pathogenic organism or skin commensal with $\geq 3$ clinical signs of infection (Table 2). Conversely, a positive blood culture is considered to be a false positive or contaminant if the organism isolated is skin commensal but there are $<3$ clinical signs of infection. 
Table 2. The definition developed by Modi et al. [4] for defining true blood stream infection in the neonatal period
(C) 2018 The Author(s). Published by S. Karger AG, Basel www.karger.com/bmh

Hamilton et al.: A Sterile Collection Bundle Intervention Reduces the Recovery of Bacteria from Neonatal Blood Culture

\author{
Increased oxygen requirement or respiratory support \\ Increased apnoeas or bradycardias \\ Hypotension \\ Glucose intolerance \\ Impaired peripheral perfusion \\ Lethargy \\ Temperature instability \\ Ileus/feed intolerance \\ Decreased urinary output \\ Metabolic acidosis
}

Case definition of neonatal blood stream infection: blood culture yielding a recognised pathogen in pure culture or a mixed growth or skin commensal plus $\geq 3$ predefined clinical signs.

The clinical data and review of positive cultures was assessed independently by two medical practitioners and subsequently by a third practitioner to ensure there was no discrepancy in the allocation.

\section{Analysis}

Rates of false-positive blood cultures were unknown in the unit prior to this study. The rates of false-positive cultures were estimated, based on published literature, at 5\%. To demonstrate a reduction in the rate of false-positive blood cultures from 5 to $0.5 \%$ with the use of a sterile collection bundle with $80 \%$ power and $\alpha=0.05$, we aimed to include 160 infants before and after the introduction of the intervention. Rates of false-positive cultures in the two groups were compared using the $\mathrm{N}-1 \chi^{2}$ test to account for small numbers [18]. Subgroup analysis was undertaken for babies $<1,500 \mathrm{~g}$ and babies $<28$ weeks' gestation. The number of unnecessary antibiotic days was also assessed and defined as the number of extra days of antibiotics received after a false-positive blood culture, compared to the number which would have been given if the blood culture had demonstrated no growth. All analyses were conducted using SPSS (Brooklyn, NY, USA). A $p$ value of $<0.05$ was taken as the level of statistical significance.

\section{Results}

During the study period (October to November 2012 and March to April 2013), data from a total of 294 babies were included in the analysis. In the PRE group, a total of 197 blood cultures were taken from 161 babies. In the POST group, 170 cultures were taken from 133 babies. The demographics of each group are outlined in Table 3.

There was a higher rate of false-positive cultures in the PRE group (9/197 [4.6\%]) compared to the POST group (1/170 [0.6\%], $p<0.05)$. A subgroup analysis was performed on infants $<28$ weeks. False-positive blood culture rates decreased from $16 \%(4 / 25)$ in the PRE group to $0 \%(0 / 30)$ in the POST group $(p<0.05)$. The $<1,500 \mathrm{~g}$ subgroup saw a decrease from $9.1 \%(4 / 44)$ in the PRE group to $0 \%(0 / 42)$ in the POST group $(p=0.05)$.

Positive cultures were identified in 16/197 (8.1\%) in the PRE group and in 9/170 (5.3\%) in the POST group ( $p=0.3$ ). Comparing the two groups, the reduction in total number of positive cultures was mainly due to the reduction in growth of CoNS $(12 / 197$ [6\%] vs. 3/190 [1.8\%], $p=0.02$ ). 
Table 3. Demographic data of patient population, frequency, and timing of blood culture

\begin{tabular}{lcc}
\hline & $\begin{array}{l}\text { Pre-intervention } \\
\text { group }\end{array}$ & $\begin{array}{l}\text { Post-intervention } \\
\text { group }\end{array}$ \\
\hline Number of infants & 160 & 133 \\
Number of blood cultures & 197 & 170 \\
Median day of life at blood culture (range) & $0(0-119)$ & $0(0-224)$ \\
Median weight (IQR), g & $2,750(1,515-3,555)$ & $2,670(1,525-3,510)$ \\
Median gestation (IQR), weeks & $37(31.7-40.1)$ & $37.14(30.2-40.1)$ \\
Number of cultures in infants $<1,500$ g and $<28$ weeks & $44(25 \%)$ & $42(20 \%)$ \\
\hline
\end{tabular}

For patients with positive cultures in the PRE group, a total of 75 days of antibiotics were given but $27(36 \%)$ of these days were considered unnecessary. In the POST group, there were 81 days of antibiotics given in total, but none given unnecessarily when analysed with the true blood stream infection criteria $(p<0.01)$.

In the follow-up comparison (June to July 2017), 167 blood cultures were obtained from 149 babies. During this time period, $7 / 167$ (4.2\%) blood cultures were positive. Two out of $167(1.2 \%)$ blood cultures were considered to be false positive ( $p=0.06$, compared with the original PRE group). Infants $<1,500 \mathrm{~g}$ had no false-positive blood cultures. One day of antibiotics was considered unnecessary.

\section{Discussion}

This study demonstrates a reduction in false-positive blood cultures between the control and intervention period. From the presented data there appears to be a sustained effect following the intervention. Our study has shown that by using a blood culture collection bundle intervention, false-positive blood cultures may be reduced. As a result, clinical staff can be more confident that a positive blood culture result equates to a true blood stream infection. This may also help reduce unnecessary antibiotic administration.

Our results concur with those found in a similar study in the setting of a paediatric emergency department. Hall et al. [11] introduced a modified sterile collection technique via a peripheral intravenous catheter and found a significant reduction in contamination rates from 3.9 to $1.6 \%$.

As this intervention is a bundle of measures, there is uncertainty as to which individual component had the greatest impact on the rates of contaminated blood cultures. A previous study by Viagappan and Kelsey [19] isolated the same species of bacteria from the culture and the patient's own skin, suggesting that in the majority of cases the bacterial contaminant comes from the patient's skin rather than the clinician. If this is the case in neonates, we could speculate that the $2 \%$ chlorhexidine was the item that had the most impact on the contamination rates.

The published definition used to determine false-positive cultures was developed by Modi et al. [4]. Their system was validated for use in infants $\leq 31$ weeks' gestation with suspected late-onset neonatal infection. We extrapolated the definition for use in infants of all gestations and potential sepsis type. This scoring system combines identified bacteria and clinical features of infection to determine true infection. This study included babies with potential early- and late-onset infection. There is currently no specific definition for earlyonset infection in newborn infants. The National Institute of Health and Care Excellence (NICE) [20] issued guidelines for using antibiotics for early-onset neonatal infection which 
suggest a series of signs in infants that may indicate an infection in term newborns. The definition used by Modi et al. [4] has a similar list of clinical signs for a preterm population. This definition is a close approximation of infection in term infants and is considered by the authors as an acceptable, if not an explicitly validated, extension of the definition.

There are some limitations to this study. Data collection relied on accurate documentation of clinical status to ascertain which clinical criteria were present. However, a comprehensive review of medical, nursing, and electronic charts provided a robust and holistic view of the clinical status. Although the advised volume of blood for culture was $1 \mathrm{~mL}$, this was not monitored and a smaller volume may potentially result in decreased yield of positive culture results and an increase in false negatives. Both potential confounding factors would be present in both cohorts and therefore not influence the results overall.

The reduction of false-positive blood cultures has potential benefits for both the patient and hospital. These benefits include reduced need further blood tests, equipment for administration of antibiotics, medical and nursing staff time as well as laboratory and drug costs. In addition, the prescription of antibiotics is restricted to those cases that genuinely require them.

Multidrug-resistant organisms are becoming more common and the responsible prescription of antibiotics is important to minimise the spread of antibiotic resistance [21, 22]. Use of antibiotics has been associated with altered gut flora $[23,24]$ and an increased risk of necrotising enterocolitis in infants [25-27]. Infants are also at risk of the ototoxic and nephrotoxic side effects of gentamicin [28] and vancomycin [29,30]. The earlier cessation of antibiotics may promote better antibiotic usage.

\section{Conclusion}

Intervention of a sterile collection bundle reduced the number of false-positive blood culture results in the setting of a neonatal intensive care unit. This in turn may reduce unnecessary antibiotic use and its associated implications.

\section{Acknowledgements}

The authors would like to recognize the contributions of the staff of St. Michael's Hospital, Regional Neonatal Intensive Care Unit.

\section{Disclosure Statement}

The intervention in this study was approved by the University Hospitals Bristol NHS Foundation Trust Departmental Governance committee. The authors have nothing to declare. This study received no specific funding and there the authors have no financial interests to declare. 


\section{References}

1 Vergnano S, Menson E, Kennea N, Embleton N, Russell AB, Watts T, et al: Neonatal infections in England: the NeonIN Surveillance Network. Arch Dis Child Fetal Neonatal Ed 2011;96:F9-F14.

2 Mitha A, Foix-L'Hélias L, Arnaud C, Marret S, Vieux R, Aujard Y, et al: Neonatal infection and 5-year neurodevelopmental outcome of very preterm infants. Pediatrics 2013;132:e372-e380.

-3 Hall KK, Lyman JA: Updated review of blood culture contamination. Clin Microbiol Rev 2006;19:788-802.

-4 Modi N, Doré CJ, Saraswatula A, Richards M, Bamford KB, Coello R, et al: A case definition for national and international neonatal bloodstream infection surveillance. Arch Dis Child Fetal Neonatal Ed 2009;94:F8-F12.

5 Isaacs D; Australasian Study Group for Neonatal Infections: A ten year, multicentre study of coagulase negative staphylococcal infections in Australasian neonatal units. Arch Dis Child Fetal Neonatal Ed 2003;88:F89-F93.

6 Huang SY, Tang RB, Chen SJ, Chung RL: Coagulase-negative staphylococcal bacteremia in critically ill children: risk factors and antimicrobial susceptibility. J Microbiol Immunol Infect 2003;36:51-55.

7 Souvenir D, Anderson DE, Palpant S, Mroch H, Askin S, Anderson J, et al: Blood cultures positive for coagulasenegative staphylococci: antisepsis, pseudobacteremia, and therapy of patients. J Clin Microbiol 1998;36: 1923-1926.

8 Thuler LC, Jenicek M, Turgeon JP, Rivard M, Lebel P, Lebel MH: Impact of a false positive blood culture result on the management of febrile children. Pediatr Infect Dis J 1997;16:846-851.

-9 McLaughlin LM, Inglis GDT, Hoellering AB, Davies MW: Relationship between blood culture collection method and proportion of contaminated cultures in neonates. J Paediatr Child Health 2013;49:105-108.

10 Silva HL, Strabelli TM, Cunha ER, Neres SF, Camargo LF, Uip DE: Nosocomial negative Staphylococci bacteremia: five year prospective data collection. Braz J Infect Dis 2000;4:271-274.

11 Hall RT, Domenico HJ, Self WH, Hain PD: Reducing the blood culture contamination rate in a pediatric emergency department and subsequent cost savings. Pediatrics 2013;131:e292-e297.

12 Adams D, Quayum M, Worthington T, Lambert P, Elliott T: Evaluation of a $2 \%$ chlorhexidine gluconate in $70 \%$ isopropyl alcohol skin disinfectant. J Hosp Infect 2005;61:287-290.

13 Gander RM, Byrd L, DeCrescenzo M, Hirany S, Bowen M, Baughman J: Impact of blood cultures drawn by phlebotomy on contamination rates and health care costs in a hospital emergency department. J Clin Microbiol 2009;47:1021-1024.

14 Bamber AI, Cunniffe JG, Nayar D, Ganguly R, Falconer E: Effectiveness of introducing blood culture collection packs to reduce contamination rates. Br J Biomed Sci 2009;66:6-9.

15 Alahmadi YM, McElnay JC, Kearney MP, Aldeyab MA, Magee FA, Hanley J, et al: Tackling the problem of blood culture contamination in the intensive care unit using an educational intervention. Epidemiol Infect 2015;143: 1964-1971.

16 Sharek PJ, Benitz WE, Abel NJ, Freeburn MJ, Mayer ML, Bergman DA: Effect of an evidence-based hand washing policy on hand washing rates and false-positive coagulase negative staphylococcus blood and cerebrospinal fluid culture rates in a level III NICU. J Perinatol 2002;22:137-143.

17 Polin RA: Management of neonates with suspected or proven early-onset bacterial sepsis. Pediatrics 2012; 129:1006-1015.

18 Campbell I: Chi-squared and Fisher-Irwin tests of two-by-two tables with small sample recommendations. Stat Med 2007;26:3661-3675.

19 Viagappan M, Kelsey MC: The origin of coagulase-negative staphylococci isolated from blood cultures. J Hosp Infect 1995;30:217-223.

20 Antibiotics for Early-Onset Neonatal Infection. CG149. London, National Institute for Health and Clinical Excellence, 2012.

21 Isaacs D: Unnatural selection: reducing antibiotic resistance in neonatal units. Arch Dis Child Fetal Neonatal Ed 2006;91:F72-F74.

22 Turnidge J, Christiansen K: Antibiotic use and resistance - proving the obvious. Lancet 2005;365:548-549.

23 Russell AB, Sharland M, Heath PT: Improving antibiotic prescribing in neonatal units: time to act. Arch Dis Child Fetal Neonatal Ed 2012;97:F141-F146.

24 Tripathi N, Cotten CM, Smith PB: Antibiotic use and misuse in the neonatal intensive care unit. Clin Perinatol 2012;39:61-68.

25 Cotten CM, Taylor S, Stoll B, Goldberg RN, Hansen NI, Sánchez PJ, et al: Prolonged duration of initial empirical antibiotic treatment is associated with increased rates of necrotizing enterocolitis and death for extremely low birth weight infants. Pediatrics 2009;123:58-66.

-26 Alexander VN, Northrup V, Bizzarro MJ: Antibiotic exposure in the newborn intensive care unit and the risk of necrotizing enterocolitis. J Pediatr 2011;159:392-397.

27 Madan JC, Farzan SF, Hibberd PL, Karagas MR: Normal neonatal microbiome variation in relation to environmental factors, infection and allergy. Curr Opin Pediatr 2012;24:753-759.

28 Rao SC, Ahmed M, Hagan R: One dose per day compared to multiple doses per day of gentamicin for treatment of suspected or proven sepsis in neonates. Cochrane Database Syst Rev 2006;1:CD005091.

29 Elyasi S, Khalili H, Dashti-Khavidaki S, Mohammadpour A: Vancomycin-induced nephrotoxicity: mechanism, incidence, risk factors and special populations. A literature review. Eur J Clin Pharmacol 2012;68:1243-1255.

-30 Vella-Brincat JWA, Begg EJ, Robertshawe BJ, Lynn AM, Borrie TL, Darlow BA: Are gentamicin and/or vancomycin associated with ototoxicity in the neonate? A retrospective audit. Neonatology 2011;100:186-193. 